One aspect of research on primate social organisation which seems to be relatively underdeveloped is that of communication, which was the subject of a review lecture by Peter Marler (Rockefeller University). It is still not known for certain whether the dozen or so sounds that make up the communicative repertoire of most monkeys and apes are ever used in the way that words are used to make sentencesone sound retaining the same meaning in different sound combinations-or whether it is always the complete pattern that conveys the message, and not the sum of its parts. It is almost certainly the entire sound combination, but the precise interpretation of primate calls in their natural context can be extremely difficult and Marler took the view that the effects of playing back natural calls should be very illuminating, perhaps especially in the laboratory in combination with single-unit recordings from the animals' cortex. (Neurophysiologists, who have had more experience with the problems of interpreting the calls of single units in their natural surroundings, might take issue with him on the latter point.)

What apes have certainly not done is to develop a language in the sense that man has, and in spite of the research resources poured into the attempt, they have not been conclusively shown to be able to use ours in a strictly linguistic way. Although D. M. Rumbaugh (Yerkes Primate Center, Atlanta) protests the contrary, David Premack (University of Pennsylvania) nct only conceded that apes never have grasped human language, but is willing to predict that they never will.

They can, however, fairly readily learn to use abstract symbols to represent objects and actions, and they can use the symbols in strings. What is missing is the evidence that the strings are composed according to syntactic rules.

Tired of trying to satisfy professional linguists that ape use of symbols fulfils the criteria for linguistic competence, some researchers have declared they would be satisfied by a "Turing test": if a man cannot tell whether he is conversing with an ape or another man, then the ape is using language. P. A. M. Seuren, collaborating with G. Ettlinger and others at the Institute of Psychiatry (London University), has, as a linguist himself, suggested a more sophisticated test which hinges on an anomaly of human usage. We say, for instance, "Don't do this and don't do that" or "Don't do this or that", but never the logically equivalent "don't do this and that". That linguistic quirk, according to Seuren, is virtually universal in human languages. The question is, would it crop up spontaneously in ape usage? The answer will not be available until Ettlinger and his colleagues have succeeded in teaching apes to use "or", which has so far not been done.

\section{Heavy ions at Caen}

from $P$. E. Hodgson

The European Physical Society Conference on Nuclear Physics with Heavy Ions was held at Caen on September 6-10, 1976.

Heavy ion physics has developed rapidly over the last few years and the conference provided an opportunity to survey the areas that are already quite well understood, and also to discuss the extensive new data on new phenomena, and the theories that have been suggested to account for them. Caen was chosen for the Conference because a powerful new heavy ion accelerator GANIL is to be built there in the next few years.

Glancing collisions between heavy ions, in which one or two ions are transferred, are useful probes of nuclear structure, and the present state of this work was surveyed by $\mathrm{O}$. Hansen and J. Garrett (University of Copenhagen). Detailed theories of these reactions have been developed, and very complicated theoretical calculations, involving the solution of coupled Schrodinger equations, are needed to analyse the data. K. Low (CEN, Saclay) showed how these calculations account for the details of the reaction mechanisms. Their very complexity does, however, make it difficult to use such reactions for nuclear structure studies. This work also needs the optical potentiaks that represent the interaction between the ions, and some of the latest methods of calculating these potentials were surveyed by D. Brink (University of Oxford).

Extremely complicated reactions take place when the heavy ions collide more nearly head-on. They can fuse together to form a highly excited compound system which then decays by particle emission (discussed by $\mathbf{M}$. Lefort (Orsay)). If the collision is intermediate between glancing and head-on the two nuclei can orbit round each other and exchange a few nucleons, becoming highly excited in the process, before separating again. Measurements have been made of the energies and masses of the ions after these deep inelastic interactions, and some of the results were considered by J. Galin (Orsay) and by L. G. Moretto (Berkeley). The theories that have been developed to account for these data were summarised in a comprehensive review by $W$. Nörenberg (University of Heidelberg). The earlier theories using the classical concept of friction are now being replaced by microscopic transport theories and the spreading of the energy distribution is described by the Fokker-Planck equation. This new and detailed understanding of deep inelastic scattering was one of the more important developments reported to the conference.

When two heavy ions combine after a non-central collision, the compound system has a large angular momentum. This preferential excitation of high spin states is a useful means of studying nuclear structure, and the development of our knowledge in this area was surveyed by Z. Szymanski (University of Warsaw).

A special session was devoted to the many attempts that have been made in the past few months in several laboratories to detect superheavy nuclei, after the announcement that evidence has been found for their natural occurrence in the mineral monazite (see Nature, 261, 627; 1976). Samples of monazite have been bombarded with protons and the spectra of the gamma rays subsequently emitted have been measured. Detailed chemical analyses have been made of samples of the mineral and reactions between heavy ions that might lead to their production have been studied. Monazite has been bombarded with heavy ions, and the recoils examined to see if any of them could have come from superheavy nuclei, but none of this work provided any evidence in support of their existence. This does not yet destroy the evidence offered in the original papers, as there are still several important points that require more detailed examination, but the evidence in favour of superheavy nuclei now looks decidedly weaker than it did a month ago. Work will certainly continue in this exciting field.

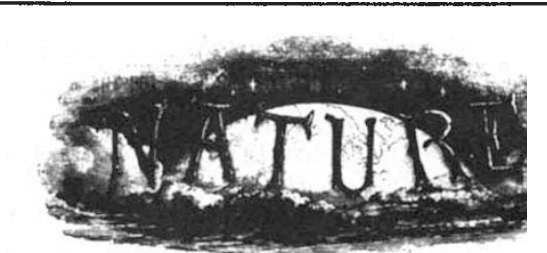

\section{A hundred years ago}

REFERRING to our recent correspondence on "Antedated Books," a correspondent calls attention to another evil practice that has of late years crept into the publishing trade, namely, that of publishing books without any date at all. Such books are generally of small literary or scientific value, but circulate among a class who are generally unable to test their value. Of course the purpose of issuing undated books is evident; works half a century old may be palmed off on the unknowing as the genuine product of the current year.

From Nature, 15, November 2, 16; 1876. 